\title{
Cyclotron resonance of a magnetic quantum dot
}

\author{
Nga T. T. Nguyen* and F. M. Peeters ${ }^{\dagger}$ \\ Departement Fysica, Universiteit Antwerpen, Groenenborgerlaan 171, B-2020 Antwerpen, Belgium
}

(Received 2 September 2008; published 11 December 2008)

\begin{abstract}
The energy spectrum of a one-electron quantum dot doped with a single magnetic ion is studied in the presence of an external magnetic field. The allowed cyclotron resonance (CR) transitions are obtained together with their oscillator strength as a function of the magnetic field, the position of the magnetic ion, and the quantum dot confinement strength. With increasing magnetic field a ferromagnetic-antiferromagnetic transition is found, which results in clear signatures in the $\mathrm{CR}$ absorption. It leads to discontinuities in the transition energies and the oscillator strengths and to an increase in the number of allowed transitions.
\end{abstract}

DOI: 10.1103/PhysRevB.78.245311

PACS number(s): 78.67.Hc, 71.55.Eq, 75.75.+a, 75.50.Pp

\section{INTRODUCTION}

Twenty years after the pioneering studies ${ }^{1}$ on magnetically doped quantum dots (QDs), both experimental and theoretical works on III-V and II-VI semiconductor systems containing a low density of magnetic ions ${ }^{2,3}$ [diluted magnetic semiconductors (DMSs)] have revealed a variety of remarkable physical properties ${ }^{4-16}$ such as, e.g., giant Zeeman splitting, spin splitting of exciton levels, magnetic polarons, etc. Recent investigations on the optical properties of II-VI manganese-doped quantum $\operatorname{dots}^{5,10-12}$ have shown that they are promising systems for, e.g., qubits. The spin of the magnetic ion (Mn ion) is used as a quantum bit, and recent experiments have shown that in single Mn-doped one-electron quantum dots it is possible to control the spin of the electron and of the magnetic ion. ${ }^{11}$ The characterization of single Mndoped few-electron II-VI quantum dots is very important. Typically, one uses interband absorption experiments. Here, we will investigate intraband transitions and show that as a function of the magnetic field different features appear when a magnetic ion is present which depend on the position of the $\mathrm{Mn}$ ion inside the QD.

Studies on $N_{e}$ strongly interacting electrons that are confined in a quantum dot and interacting with a single magnetic ion have been mostly limited to the experimental ${ }^{11}$ and theoretical studies ${ }^{10,14-16}$ of the ground state (GS) and the thermodynamic properties of dots containing a small number of electrons.

Cyclotron resonance (CR) has been an important experimental technique to investigate the properties of electrons (and holes). The advantage is that CR transitions involve only one type of carriers (electrons or holes) facilitating the interpretation of the results. CR is also often used to obtain information on the effective mass of the carriers.

Cyclotron resonance has been studied in bulk semiconductors ${ }^{17}$ and later on also in semiconductor quantum dots, ${ }^{18-20}$ where it was shown that in parabolic confined quantum dots Kohn's theorem still holds, and thus, the CR transitions are independent of the number of electrons. The latter is no longer true if the confinement potential is nonparabolic ${ }^{19}$ or when the carriers obey a nonparabolic energy spectrum.

Very recently, ${ }^{12}$ the intraband optical properties of a threedimensional (3D) single-electron CdTe/ZnTe quantum dot containing one or two Mn impurities confined by a potential that is parabolic in the $x y$ plane and has a quantum-well confinement along the $z$ axis were investigated. Different absorption lines (mixing with the lines to higher excited levels for quantum-well systems - as for example, see Ref. 19 for studies of CR in quantum-well systems without a Mn ion) were predicted as well as crossings and anticrossings of allowed CR transitions as a function of an external magnetic field.

In the present paper we calculate the single-particle states of a quasi-two-dimensional parabolic quantum dot in the presence of a single $\mathrm{Mn}$ ion and an external perpendicular magnetic field. We investigate the allowed cyclotron resonance transitions and their corresponding oscillator strength (OS). We have in mind experimental-realized systems [i.e., $\mathrm{Cd}(\mathrm{Mn}) \mathrm{Te}$ ] where the lateral size is much larger than the height of the quantum dot, and consequently the system behaves like a quasi-two-dimensional quantum dot. As in Ref. 12 we find different resonant transitions due to the electronmagnetic ion (e-Mn) spin-spin exchange interaction but we also investigate these transitions as a function of the position of the Mn ion in the quantum dot. We find crossing and anticrossing features that depend on the position of the magnetic ion in the quantum dot and on the strength of the confinement potential. We will focus on the allowed transitions and concentrate on the different CR lines that become possible due to the presence of the Mn ion. Due to the presence of the $\mathrm{Mn}$ ion the electron spin will not always be parallel to the external magnetic field. It will, together with the Mn ion, define two kinds of relative orientation of their spins called ferromagnetic (FM) and antiferromagnetic (AFM). The FMAFM transition can be influenced by changing the exchange interaction parameters which we will realize by moving the magnetic ion to different positions. We show that this can change the position and the number of crossing and anticrossing points and consequently influence the CR lines. We find that the CR transitions now contain information not only on the electron state but also on the magnetic-ion-electron interaction and the magnetic state of the magnetic ion. Because of the rich physics involved we will limit our discussion here to the fundamental single-electron quantum dot case.

This paper is organized as follows. Section II introduces the model and the numerical method. In Sec. III we present our numerical results for the energy spectrum. Section IV is 
devoted to the cyclotron resonance transitions where we calculate the oscillator strength and the allowed transition energy spectrum. Our discussion and conclusions are presented in Sec. V.

\section{THEORETICAL MODEL}

A quantum dot containing a single electron with spin $\vec{s}$ confined by a parabolic potential and interacting with a single magnetic ion $\left(\mathrm{Mn}^{2+}\right)$ with spin $\vec{M}$ and a magnetic field is described by the following Hamiltonian:

$$
\begin{aligned}
\hat{H}= & \left\{\frac{1}{2 m^{*}}\left[-i \hbar \vec{\nabla}_{\vec{r}}+e \vec{A}(\vec{r})\right]^{2}+\frac{1}{2} m^{*} \omega_{0}^{2} \vec{r}^{2}\right\} \\
& +\frac{1}{2} \hbar \omega_{c}\left(g_{e} m^{*} s_{z}+g_{\mathrm{Mn}} m^{*} M_{z}\right)-J_{c} \vec{s} \cdot \vec{M} \delta(\vec{r}-\vec{R}) .
\end{aligned}
$$

The first two terms are, respectively, the single-particle kinetic energy and the confinement potential for the electron. The third and the fourth terms are the electron and magneticion Zeeman energies, respectively. The last term is the electron $\mathrm{Mn}$ spin-spin exchange interaction with strength $J_{c}$. The vector potential is taken in the symmetric gauge $\vec{A}=B / 2(-y, x, 0)$ where the magnetic field $\vec{B}$ is perpendicular to the plane of the interface. The confinement frequency $\omega_{0}$ defines the confinement length $l_{0}=\sqrt{\hbar / m^{*} \omega_{0}} \cdot g_{e}$ and $g_{\mathrm{Mn}}$ are the Landé $g$ factors of the host semiconductor and the magnetic ion, respectively. We introduce a dimensionless parameter $S_{C}=\left(a_{B}^{*} / l_{0}\right)^{2}$ called the confinement strength (square comes from the fact that $\left.\omega_{0} \sim 1 / l_{0}^{2}\right)$. In a many-electron system $S_{C}$ is $S_{C}=1 / \lambda_{C}^{2}$ with $\lambda_{C}=l_{0} / a_{B}^{*}$ as the Coulomb interaction strength. ${ }^{15} a_{B}^{*}=4 \pi \epsilon_{0} \epsilon \hbar^{2} / m^{*} e^{2}$ is the effective Bohr radius. The cyclotron frequency is $\omega_{c}=e B / m^{*}$. We use the set of parameters ${ }^{14}$ that is applicable to a self-assembled Cd(Mn)Te quantum dot with a typical lateral size of about tens of nanometers. The dielectric constant $\epsilon=10.6$, effective mass $m^{*}=0.106 m_{0}, a_{B}^{*}=52.9 \AA, g_{e}=-1.67, g_{\mathrm{Mn}}=2.02, J_{c}$ $=1.5 \times 10^{3} \mathrm{meV} \AA^{2}$, and $l_{0}$ is about tens of angstroms $\left(\hbar \omega_{0}\right.$ corresponding to tens of $\mathrm{meV})$. For example, with $\hbar \omega_{0}$ $=51.32 \mathrm{meV}$ gives $l_{0}=26.45 \AA$ and corresponds to $S_{C}=4$; $\hbar \omega_{0}=12.83 \mathrm{meV}$ gives $l_{0}=52.9 \AA$ and corresponds to $S_{C}$ $=1$.

We use the single-particle states in a parabolic confinement potential, namely, the complete basis of Fock-Darwin (FD) orbitals $\phi_{n l}(\vec{r})$ and spin functions $\chi_{\sigma}(\vec{s})$,

$$
\phi_{n l \sigma}(\vec{r}, \vec{s})=\varphi_{n l}(\vec{r}) \chi_{\sigma}(\vec{s}),
$$

with the Fock-Darwin orbitals

$$
\varphi_{n l}(\vec{r})=\frac{1}{l_{H}} \sqrt{\frac{n !}{\pi(n+|l|) !}}\left(\frac{r}{l_{H}}\right)^{|l|} e^{-i l \theta} e^{-r^{2} / 2 l_{H}^{2}} L_{n}^{|l|}\left(\frac{r^{2}}{l_{H}^{2}}\right) .
$$

The single-particle (spin $\sigma$ ) Fock-Darwin orbital energy in the presence of a magnetic field at site $i=\{n, l\}$ is given by

$$
E_{i, \sigma}=\hbar \omega_{H}(2 n+|l|+1)-\hbar \omega_{c} l / 2,
$$

with $\omega_{H}=\omega_{0} \sqrt{1+\left(\omega_{c} / 2 \omega_{0}\right)^{2}}$ that can also be expressed in terms of a new length $l_{H}, \omega_{H}=\hbar / m^{*} l_{H}^{2}$.
We can now rewrite the Hamiltonian in second-quantized form,

$$
\begin{aligned}
\hat{H}= & \sum_{i, \sigma} E_{i, \sigma} c_{i, \sigma}^{+} c_{i, \sigma}+\frac{1}{2} \hbar \omega_{c}\left(g_{e} m^{*} s_{z}+g_{\mathrm{Mn}} m^{*} M_{z}\right)-\sum_{i j} \frac{1}{2} J_{i j}(\vec{R}) \\
& \times\left[\left(c_{i, \uparrow}^{+} c_{j, \uparrow}-c_{i, \downarrow}^{+} c_{j, \downarrow}\right) M_{z}+c_{i, \uparrow}^{+} c_{j, \downarrow} M^{-}+c_{i, \downarrow}^{+} c_{j, \uparrow} M^{+}\right]
\end{aligned}
$$

where $i$ denotes a set of quantum numbers $\{n, l\} . L_{z}, s_{z}$, and $M_{z}$ are the projections of the angular momentum of the electron, its spin, and spin of the magnetic ion in the direction of the magnetic field, respectively. $M_{z}, M^{+}$, and $M^{-}$are the $z$ projection, raising operator, and lowering operator of the magnetic-ion spin, respectively. The e-Mn interaction appearing in this form describes the conservation of the electron spin in the e-Mn interaction of, e.g., two configurations $i$ and $j$ in the first term, and the spin exchange of these configurations by flipping the electron spin at one site along with a compensation by flipping the Mn-ion spin at the other site, and vice versa, in the last two terms. This interaction parameter is

$$
J_{i j}(\vec{R})=J_{c} \varphi_{i}^{*}(\vec{R}) \varphi_{j}(\vec{R}),
$$

which is a product of the two Fock-Darwin orbitals of states $i$ and $j$ calculated at the position of the magnetic ion $R_{\mathrm{Mn}}$.

We construct the many-particle wave function using the configuration-interaction (CI) method,

$$
\Psi\left(\vec{x}^{*}, \vec{M}\right)=\sum_{k=1}^{N_{c}} c_{k} \Psi_{k}\left(\vec{x}^{*}, \vec{M}\right),
$$

where $\vec{x}^{*}$ is the radial and spin coordinate of the electron and $\Psi_{k}$ is the $k$ th state of the noninteracting many-electron wave function (in this case for one electron) determined by

$$
\Psi_{k} \Rightarrow|k\rangle=\left|c_{k \uparrow(\downarrow)}^{+}\right\rangle\left|M_{z}^{k}\right\rangle .
$$

$M_{z}^{k}$ runs through six states: $5 / 2,3 / 2, \ldots,-5 / 2$. The number of configurations $N_{c}=12 N_{s}$, with $N_{s}$ as the number of orbitals that are included, and is taken to be sufficiently large in order to guarantee sufficient accuracy.

\section{ENERGY SPECTRUM}

The presence of the magnetic ion leads to different arrangements of the spins of the electron and the magnetic ion depending on the strength of the magnetic field. This is due to the interplay between, on one hand, the contributions of the Zeeman energies of the electron and the magnetic ion, and on the other hand, the exchange interaction-both depend on the magnetic field. Two different arrangements of the spins of the electron and the $\mathrm{Mn}$ ion are possible: ferromagnetic and antiferromagnetic. The ferromagnetic arrangement is found for very small magnetic field when the exchange energy dominates over the Zeeman energy terms. In the reverse case the AFM arrangement of electron and magnetic-ion spins is found. As illustrated in Fig. 1, the FMAFM phase-transition line depends also on the position of the magnetic ion. Note that in nonzero magnetic field, the magnetic ion is always antiparallel to the magnetic field due to the positive value of its $g$ factor. 


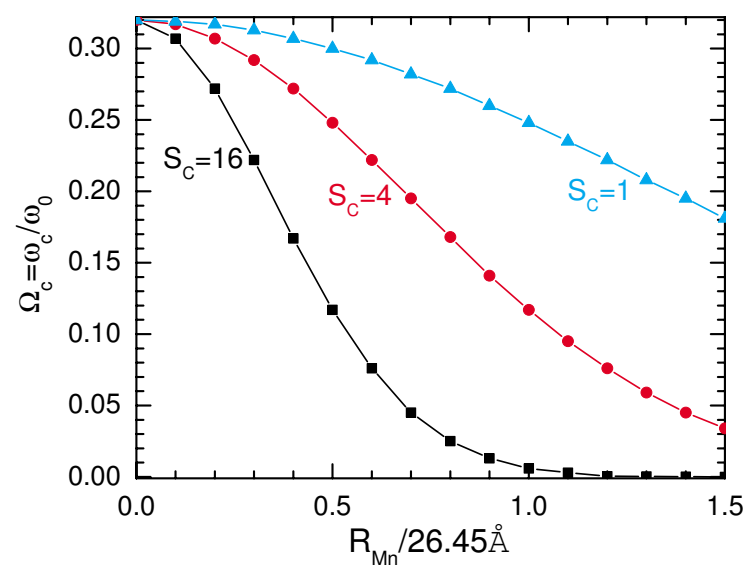

FIG. 1. (Color online) Phase diagram of $\Omega_{C}-R_{\mathrm{Mn}}$ plotted for three different confinement strengths $S_{C}$. The region below (above) the line corresponds each time to the ferromagnetic (antiferromagnetic) phase, respectively.

When the magnetic ion is located at the center of the dot, the FM-AFM transition occurs at the same magnetic field for any value of the confinement strength as is made clear in Fig. 1. The e-Mn exchange energy is maximal for a Mn ion situated in the center of the quantum dot. Note that in a oneelectron system, the electron density is maximum at the center of the dot and the electron is found in the $s$ shell where the $J_{c}$ matrix element is also found to be maximum at the center of the dot. Moving the Mn ion out of the center of the quantum dot will decrease this e-Mn exchange interaction. The electron probability at the $\mathrm{Mn}$ ion is now a strong function of the confinement strength. With decreasing e-Mn exchange energy (i.e., by moving the $\mathrm{Mn}$ ion out of the center of the QD or increasing the confinement), a smaller external magnetic field is needed to induce the FM-AFM transition, as can be seen from Fig. 1.

In the presence of the exchange energy term in Eq. (1) the GS consists of contributions from different configurations of $\left(s_{z}, M_{z}\right)$. The electron is still found in the $s$ shell even at very high magnetic field. Unlike the system without a magnetic ion where the spin of the electron always orients parallel to the magnetic field, the system containing a magnetic ion has a wave function containing contributions from states where the electron is antiparallel to the magnetic field. This leads to an attraction to the magnetic ion and reduces the total energy.

When a magnetic ion is placed inside the quantum dot the energy spectrum is modified to the one given in Fig. 2 which shows the results in the case where the $\mathrm{Mn}$ ion is situated at the center [Fig. 2(a)] and at $\left(0.5 l_{0}, 0\right)$ [Fig. 2(b)] inside the quantum dot. Note that now each $B=0$ energy level is split into many different levels having different Zeeman splittings. There are also many more crossings and anticrossings and periodic opening of energy gaps in the spectra. The position and the number of energy gaps, and consequently the crossing and anticrossing points, depend on the position of the magnetic ion as can be seen in Fig. 2, in particular, in the regions inside the blue boxes. We explicitly refer to energy levels 85 [Fig. 2(a)] and 97 [Fig. 2(b)] to highlight the differences in the energy spectrum. When changing the position of the $\mathrm{Mn}$ ion we find that some of the crossing points turn into anticrossing points and at some of the anticrossings the size of the energy gap increases. That will be shown and discussed in Sec. IV.

The $B=0$ ground-state level is split into 12 levels because it is composed of $s_{z}= \pm 1 / 2$ and $M_{z}= \pm 5 / 2, \pm 3 / 2, \pm 1 / 2$ spin states. The next $B=0$ level is the $p$-electron state, and because the electron orbital momentum is 1 this level splits into 24 levels. Note that for small magnetic fields, the states whose wave function have the $(0,1)$ or the $(0,-1)$ FockDarwin states as their largest contribution stay lower in energy as compared to, e.g., the states whose wave function have $(0,2),(1,0)$, etc. as the largest contribution. However, as the magnetic field increases, there are changes in relative position of the energy levels, i.e., whose wave function has $(0,-1)$ as the largest contribution will now be higher in energy than, e.g., the state which has $(0,2)$ as the major contributing Fock-Darwin state.

The presence of the magnetic ion leads to a mixing of the Fock-Darwin orbitals for the different eigenstates in contrast to the quantum dot without a magnetic ion, where those
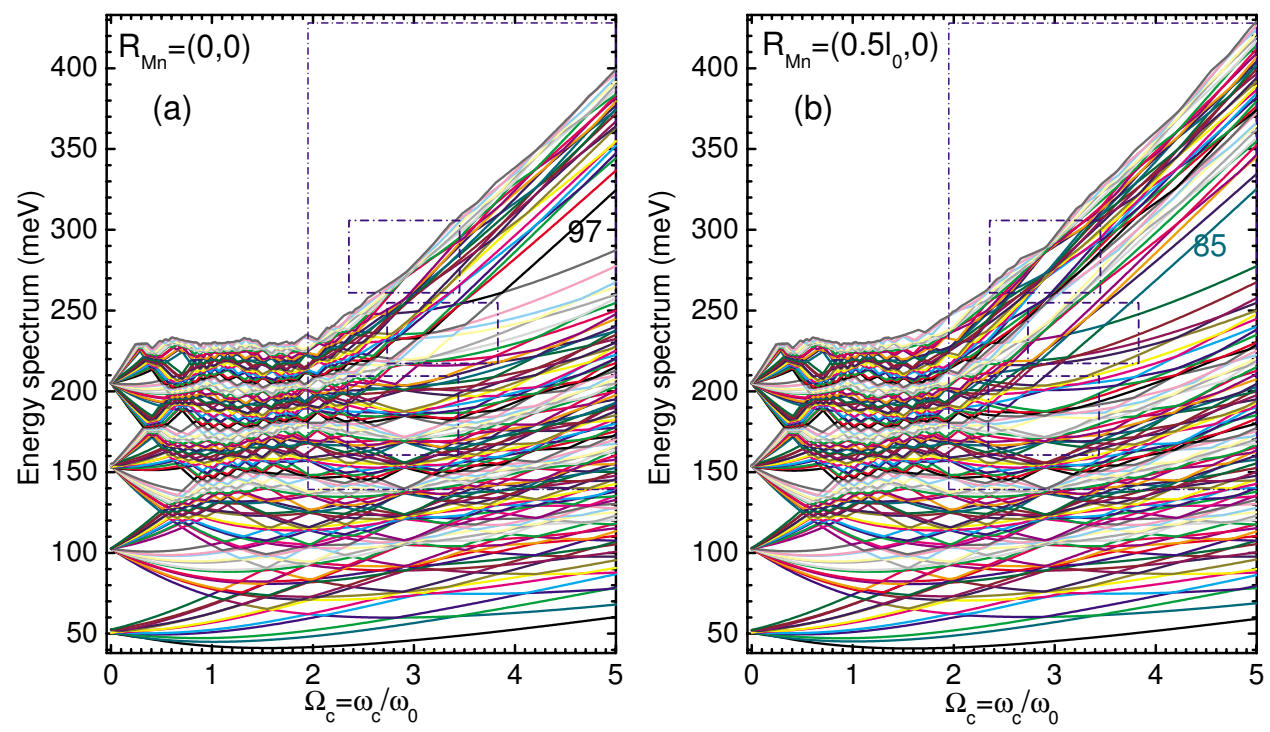

FIG. 2. (Color online) First 120 levels of the energy spectrum of a single-electron quantum dot with the magnetic ion located (a) at the center and (b) at $\left(0.5 l_{0}, 0\right)$ of the QD for confinement strength $S_{C}=4$. The colors that match the corresponding energy levels are kept the same in the two plots. The blue dash-dotted boxes highlight the regions where both figures exhibit the largest difference. 


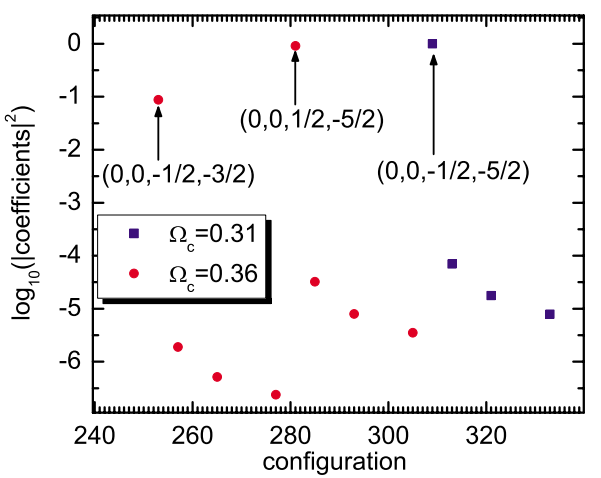

FIG. 3. (Color online) Contributions to the ground-state wave function from the different Fock-Darwin wave functions ( $x$ axis) in the case of the quantum dot of Fig. 2(a) for two different magnetic fields: $\Omega_{c}=0.31(\mathrm{FM})$ and $0.36(\mathrm{AFM})$.

Fock-Darwin orbitals are the eigenstates of the dot. We show in Fig. 3 the contribution of the different Fock-Darwin states to the wave function of the GS for two different values of the magnetic field, $\Omega_{c}=0.31$ and 0.36 . $N_{c}$ configurations that were defined in Sec. II are the $x$ axis. The configurations that contribute more than $1 \%$ are indicated by four quantum numbers $\left(n, l, s_{z}, M_{z}\right)$. At $\Omega_{c}=0.31$ where the system is in the FM phase, the GS wave function has the Fock-Darwin state $(0,0,-1 / 2,-5 / 2)$ as the largest contribution, while at AFM magnetic field $\Omega_{c}=0.36$ the two states $(0,0,-1 / 2,-3 / 2)$ and $(0,0,1 / 2,-5 / 2)$ give the dominant contribution.

\section{CYCLOTRON TRANSITIONS AND OSCILLATOR STRENGTH}

In a cyclotron resonance experiment, an electron in quantum state $i=(n, l)$ with energy $E_{i}$ is excited to a higher energy state $E_{j}\left[j=\left(n^{\prime}, l^{\prime}\right)\right]$ with transition amplitude $A_{i j}$; the associated oscillator strength for circular polarized light is ${ }^{19}$

$$
f_{i j}=\frac{2 \Delta E_{i j}}{\hbar \omega_{H}} \frac{\left|A_{i j}\right|^{2}}{l_{H}^{2}},
$$

where

$$
A_{i j}=\left\langle\Psi_{i}\left(\vec{x}^{*}, \vec{M}\right)\left|r e^{ \pm i \theta}\right| \Psi_{j}\left(\vec{x}^{*}, \vec{M}\right)\right\rangle .
$$

$\Delta E_{i j}=E_{j}-E_{i}$ is the transition energy. It is clear that the state of the magnetic ion is not altered during such a transition. The electron transition fulfills the selection rules: $\Delta l=l^{\prime}-l$ $= \pm 1 ; \Delta s_{z}=0 ;$ and $\Delta M_{z}=0$.

A general form for the wave function of state $i$ is

$$
\Psi_{i}\left(\vec{x}^{*}, \vec{M}\right)=\sum_{\alpha}^{N_{c}} c_{\alpha} \Psi_{i \alpha}\left(\vec{x}^{*}, \vec{M}\right),
$$

where $\Psi_{i \alpha}\left(\vec{x}^{*}, \vec{M}\right)$ is the single-electron Fock-Darwin solution in the presence of the magnetic ion defined via Eq. (8). Now we calculate $A_{i j}$ by integrating Eq. (10) over $\vec{r}$. The final expression for $A_{i j}$ can be written as

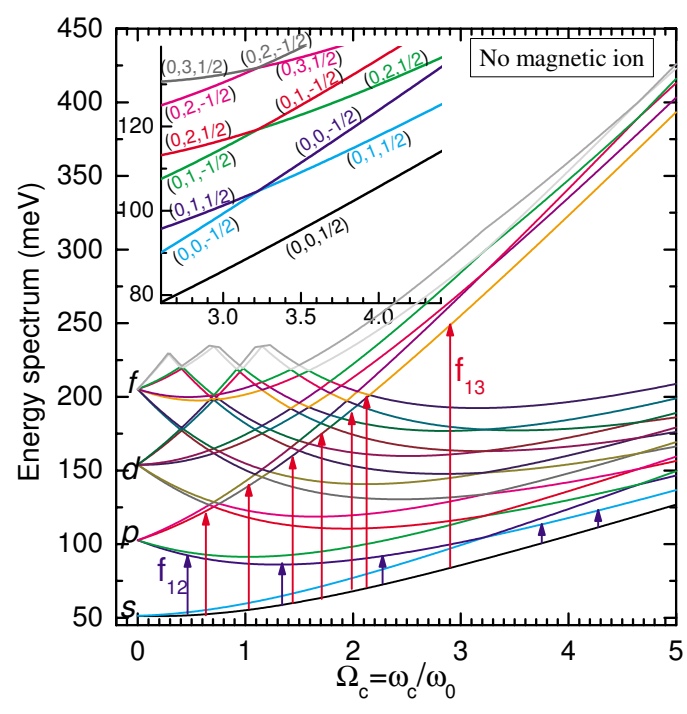

FIG. 4. (Color online) First 20 levels (up to the $f$ shell) of the energy spectrum of a one-electron quantum dot without a magnetic ion. The inset is a magnification of the region $2.6<\Omega_{c}<4.4$ that clarifies the CR transitions of the electron from the GS $(0,0,1 / 2)$ to either $(0,1,1 / 2)$ or $(0,-1,1 / 2)$ with oscillator strengths $f_{12}$ (blue) and $f_{13}$ (red), respectively. The energy levels are indicated by their quantum numbers $\left(n, l, s_{z}\right)$. The allowed transitions are indicated by the blue and red arrows.

$$
A_{i j}=\sum_{\alpha}^{N_{c}} \sum_{\beta}^{N_{c}} c_{\alpha}^{*} c_{\beta} \delta_{s_{z_{\alpha} s_{\beta}}} \delta_{M_{z_{\alpha}} M_{z_{\beta}}} A_{i j}^{\alpha \beta}
$$

where

$$
\begin{aligned}
A_{i j}^{\alpha \beta}= & \delta_{n_{\beta}, n_{\alpha}} \delta_{l_{\beta}, l_{\alpha} \pm 1} l_{H} \sqrt{n_{\alpha}+\left|l_{\alpha}\right|+1} \\
& -\delta_{n_{\beta}, n_{\alpha}+1} \delta_{l_{\beta}, l_{\alpha} \pm 1}\left(1-\delta_{l_{\alpha}, 0}\right) l_{H} \sqrt{n_{\alpha}+1}
\end{aligned}
$$

is the transition amplitude element identical to Eq. (18) of Ref. 19. The transition amplitude (12) is a sum over all possible transition amplitudes of the respective single states $\alpha$ and $\beta$.

To simplify the subsequent discussion we show in Fig. 4 first the results for the case when no magnetic ion is present. The selection rule allowed transitions (i.e., $\Delta n=0$ and $\Delta l$ $= \pm 1)$ are indicated by the vertical arrows which are transitions from the ground state $(0,0)$ to the $p$-shell $(0,1)\left(p^{+}\right)$and $(0,-1)\left(p^{-}\right)$with the respective oscillator strengths $f_{12}$ (blue) and $f_{13}$ (red). As we can see from this figure, with increasing the magnetic field, the states with positive $l$ have lower transition energy as compared to the negative $l$. This is illustrated by the blue and red arrows in Fig. 4. From this figure, for the magnetic-field range $\Omega_{c}<3.22, f_{12}$ corresponds to the transition of the electron from the ground state to the third state (level $1 \rightarrow 3$ ) while the other transition $f_{13}$ corresponds to the transition of the electron from the ground-state level to the fifth, then seventh, then ninth, then eleventh, and then thirteenth level. Note that in the limit of $\Omega_{c} \rightarrow \infty, f_{12} \rightarrow 0$.

In the presence of the magnetic ion, due to the ferromagnetic (at very small magnetic field) and antiferromagnetic (at larger magnetic field) couplings of the electron with the mag- 


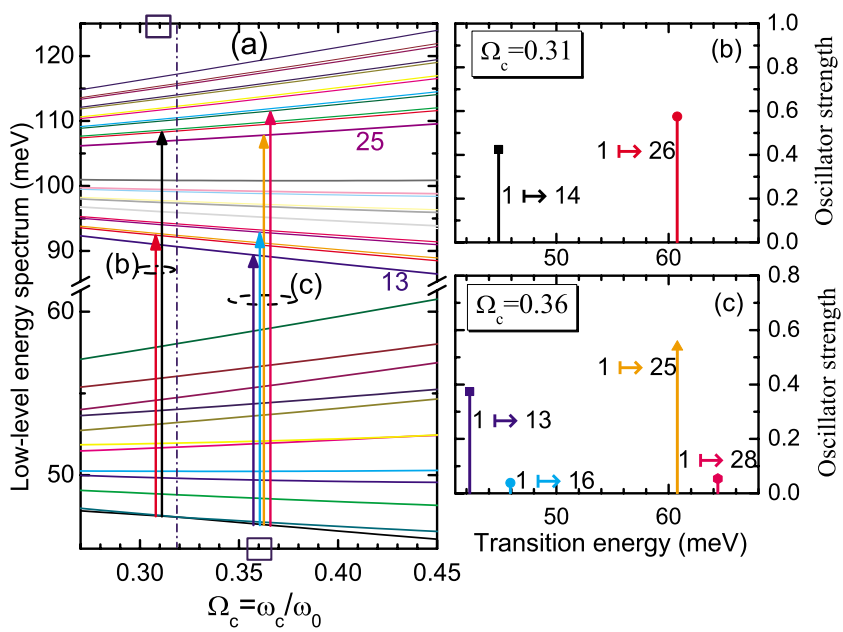

FIG. 5. (Color online) (a) Low-energy spectrum where some of the allowed transitions are indicated by arrows around the magnetic fields $\Omega_{c}=0.31$ and 0.36 . The corresponding oscillator strengths of these transitions are shown in (b) and (c). The magnetic impurity is located at the center of the dot and the confinement strength $S_{C}$ $=4$. The blue dash-dotted line at $\Omega_{c}=0.32$ indicates the ground-state FM-AFM transition.

netic ion, the energy spectrum splits into many energy levels (see Fig. 2) and no simple selection rules hold. The CR spectrum now consists of many more peaks.

We first discuss in detail the electron transition for the case where the magnetic ion is located at the center of the quantum $\operatorname{dot}\left[R_{\mathrm{Mn}}=(0,0)\right]$ for magnetic fields $\Omega_{c}=0.31$ (ferromagnetic phase) and $\Omega_{c}=0.36$ (antiferromagnetic phase-as can be seen from Fig. 1). We found two allowed transitions as seen in Fig. 5 for $\Omega_{c}=0.31$ with their respective resonant frequencies. Remember that at this magnetic field (within the region $\Omega_{c}<0.32$ ), the system is in the ferromagnetic phase where the electron and the magnetic ion are both antiparallel to the magnetic field. The transitions from the ground state (level number 1), where the electron is mostly in the $s$ orbital, to the state whose wave function has the orbital $(0,1)$ as the largest Fock-Darwin contribution correspond to the black line and to the state whose wave function has the orbital $(0,-1)$ as the largest contribution correspond to the red line, respectively. The resonances are found for transitions to the fourteenth and the twenty-sixth levels. The corresponding result for the antiferromagnetic phase are shown in Fig. 5(c) for $\Omega_{c}=0.36$. Notice that now we have four possible transitions with oscillator strength larger than $1 \%$ instead of two transitions in the previous case. This is in agreement with the effect found in Ref. 12. These four transitions correspond to the excitations of the electron from the ground state to the energy levels: thirteenth (blue square), sixteenth (cyan rhombus), twenty-fifth (orange triangle), and twenty-eighth (pink circle). The first two, blue square and cyan rhombus, belong to the transition of the electron from the $s$ ground state to the $p^{+}$state (with positive angular momentum); while the other two belong to the other transition of the electron to the $p^{-}$state (with negative angular momentum). The origin of this difference as compared to the case of $\Omega_{c}=0.31$ can also be explained by looking at the GS wave-

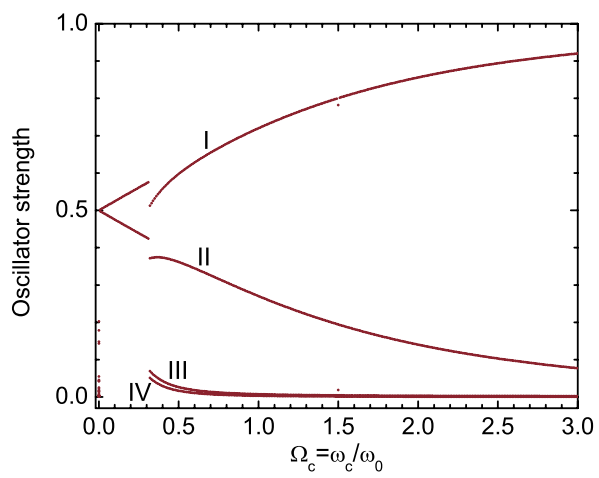

FIG. 6. (Color online) Oscillator strength of the allowed electron transitions at various magnetic fields of the same magnetic quantum dot as plotted in Fig. 5. I, II, III, and IV indicate the four branches of the OS curve as $\Omega_{c} \geq 0.32$.

function contributions of the Fock-Darwin states as shown in Fig. 3.

From Fig. 6 we find that the main transitions come from transitions to the two $p$ orbitals. The oscillator strength exhibits a discontinuity at $\Omega_{c}=0.32$ where the FM-AFM transition takes place. The wave function of the ground state changes from favoring the configurations with electron spin down to the configurations with electron spin up as can be seen from Fig. 3. As the magnetic field increases from $\Omega_{c}$ $=0.31$ where the system is in the FM phase to $\Omega_{c}=0.36$ where the system is in the AFM phase, the largest FockDarwin contribution to the GS wave function goes from $\left(n, l, s_{z}, M_{z}\right)=(0,0,-1 / 2,-5 / 2)$ to $(0,0,-1 / 2,-3 / 2)$ and $(0,0,1 / 2,-5 / 2)$. Consequently, the final state of the electron transition shifts. We will clarify this point in Fig. 7 by using the black arrows to direct the attention of the reader to the final states for the transitions of the electron. For example, for $\Omega_{c}<0.32$, the nonzero resonances are the lines from the ground state to the fourteenth and the twenty-sixth levels.

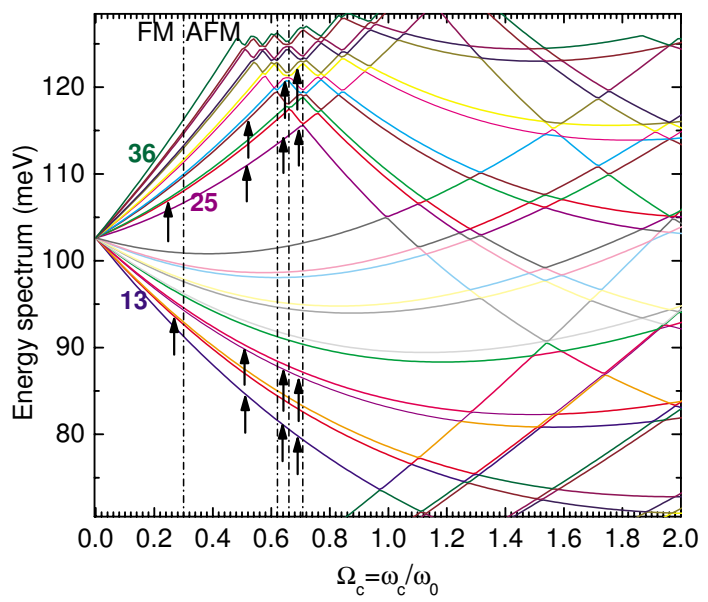

FIG. 7. (Color online) Low-level energy spectrum of the same quantum dot as in Fig. 5 is plotted from the thirteenth to thirty-sixth levels to indicate the final states of the electron transition. The black arrows indicate the specific energy levels as the final state of the transition. The final states shift following the arrows as the magnetic field increases. The numbers 13, 25, and 36 are the level numbers of the edge of subparts of the spectrum. 


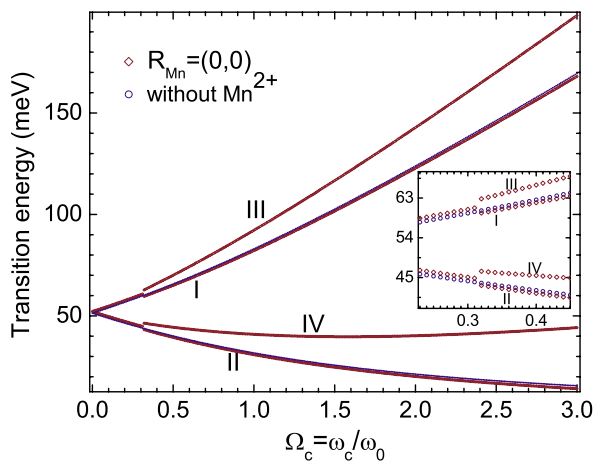

FIG. 8. (Color online) Transition energy for the same quantum dot as plotted in Fig. 6 with four branches I, II, III, and IV corresponding to the four branches in the OS curve. Blue curves are the transition energy in the absence of a magnetic ion. The inset is a magnification around the FM-AFM transition magnetic field.

When the system transits to the AFM phase, the number of resonance lines increases from two to four. In the region $0.32 \leq \Omega_{c}<0.62$, the transitions are to the thirteenth and sixteenth levels, the twenty-fifth and twenty-eighth, and the main contributions are the lines to the thirteenth, the twentyfifth, etc. This is explained as follows. For the FM magneticfield range $\Omega_{c}<0.32$, the electron and the $\mathrm{Mn}$ ion are both antiparallel to the magnetic field and $s_{z}+M_{z}=-3$ with the configuration of $\left(n=0, l=0, s_{z}=-1 / 2, M_{z}=-5 / 2\right)$ having the probability of almost unity. This is due to the fact that the commutator of the $z$ component of the total spin commutes with the Hamiltonian, $\left[s_{z}+M_{z}, H\right]=0$. Beyond this magneticfield region, $\Omega_{c} \geq 0.32$, the electron and the $\mathrm{Mn}$ ion are antiparallel, and since the Zeeman (spin) part of the Mn ion is always larger than that of the electron and the exchange energy becomes the smallest one among these three competing energies, there are two configurations $(0,0,1 / 2,-5 / 2)$ and $(0,0,-1 / 2,-3 / 2)$ with $s_{z}+M_{z}=-2$ of the GS wave function

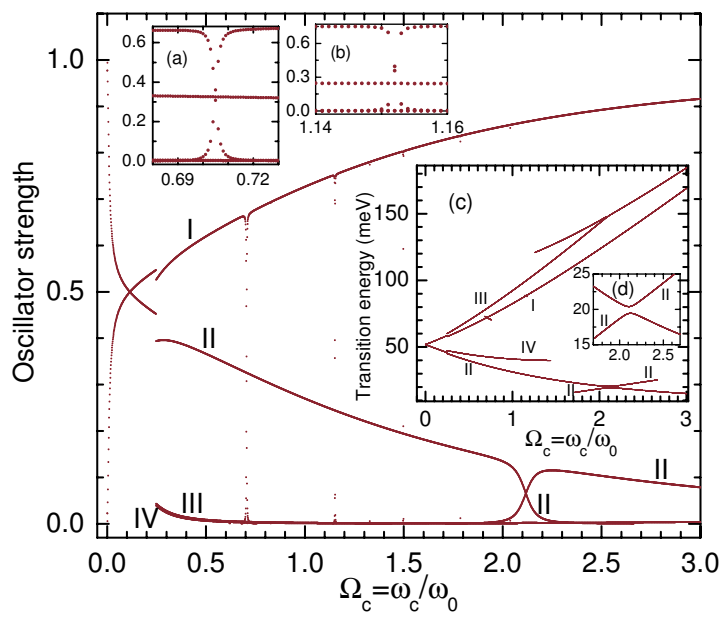

FIG. 9. (Color online) Oscillator strength with two magnifications around (a) $\Omega_{c}=0.71$ and (b) $\Omega_{c}=1.15$, and (c) transition energy with a magnification around (d) $\Omega_{c}=2.12$ for the same quantum dot as plotted in Fig. 6, but the magnetic ion is located at $\left(0.5 l_{0}, 0\right)$. I, II, III, and IV indicate the four branches when $\Omega_{c}$ $\geq 0.25$ in the OS and the corresponding transition energies. as the main contributions. It is obvious that the exchange energy now has the largest contribution coming from the second term [corresponding to configuration $(0,0,1 / 2$, $-5 / 2)]$ and the second largest from the last term [corresponding to configuration $(0,0,-1 / 2,-3 / 2)]$ in the last sum in Eq. (5). As the system is in the AFM, the final states of the major parts in the OS that are the branches I and II in Fig. 6 and the corresponding transition energies in Fig. 8 have wave functions that are the FD states $(0,-1,1 / 2,-5 / 2)$ and $(0,1,1 / 2,-5 / 2)$, respectively, as the major contributions. The other two transitions (III and IV) come from the spinspin exchange interactions corresponding to the final states that have wave functions containing FD states $(0,-1$, $-1 / 2,-3 / 2)$ and $(0,1,-1 / 2,-3 / 2)$, respectively, as their major contributions. In general, the largest one among all the small contributions can reach to about $5 \%-10 \%$ of the total OS. With increasing magnetic field, these smaller contributions to the OS decrease to zero.

The oscillator strength for the case where the magnetic ion is located at the center of the dot samples the central region of the quantum dot. In this situation the magnetic ion does not interact with the $p$ orbitals. The four branches in the OS (Fig. 6) and transition energy (Fig. 8) in the AFM phase are smooth with respect to the magnetic field. Moving the $\mathrm{Mn}$ ion to other positions away from the center of the dot


FIG. 10. (Color online) Magnification of the low-level spectrum of Fig. 2 for the cases where the magnetic ion is located at the center and at $\left(0.5 l_{0}, 0\right)$ in the quantum dot. The black arrows indicate how the final state moves with magnetic field for the lowestenergy CR transition. 

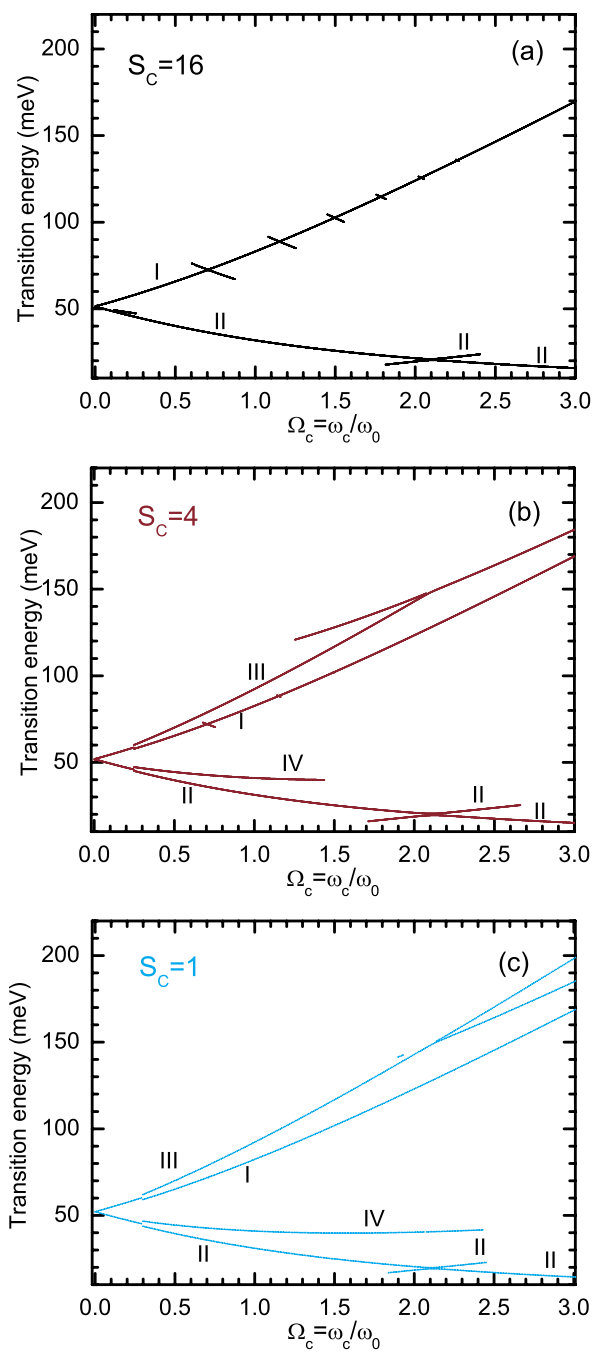

FIG. 11. (Color online) Transition energy for the quantum dot with the magnetic ion located at $\left(0.5 l_{0}, 0\right)$ and for three different values of $S_{C}$. I, II, III, and IV indicate the four [two in (a)] main branches when the system is in the AFM phase. The transition energy is scaled by the ratio of $S_{C}$ to $S_{C}=4$.

gives rise to the interactions of the $\mathrm{Mn}$ ion with the $p$ orbitals, and this is expected to change the OS by the changes in the two main branches (I and II in Figs. 6 and 8). The influence of the position of the magnetic ion on the CR transitions is shown in Fig. 9 for the case where the Mn ion is located, e.g., at $\left(0.5 l_{0}, 0\right)$. First of all, we notice a discontinuity of the oscillator strength at $\Omega_{c}=0.25$ which corresponds to the FMAFM transition. Second, we find an unusual behavior at $\Omega_{c}$ $=2.12$ where the lower curve exhibits a crossing. This is a consequence of the existence of an energy gap between the fourth and the fifth levels at $\Omega_{c}=2.12$ as is clearly shown in Fig. 10(b) for the case where the $\mathrm{Mn}$ ion is located at $\left(0.5 l_{0}, 0\right)$. These fourth and fifth levels have the Fock-Darwin states $(0,1,1 / 2,-5 / 2)$ and $(0,0,-1 / 2,-3 / 2)$ as their major contributions. When the $\mathrm{Mn}$ ion is located at $\left(0.5 l_{0}, 0\right)$ the exchange matrix elements between the $p$ orbitals are nonzero which leads to the anticrossing. This is the reason why the corresponding transition energy [see Figs. 9(c) and 9(d)] exhibits an anticrossing behavior at $\Omega_{c}=2.12$. The energy gap
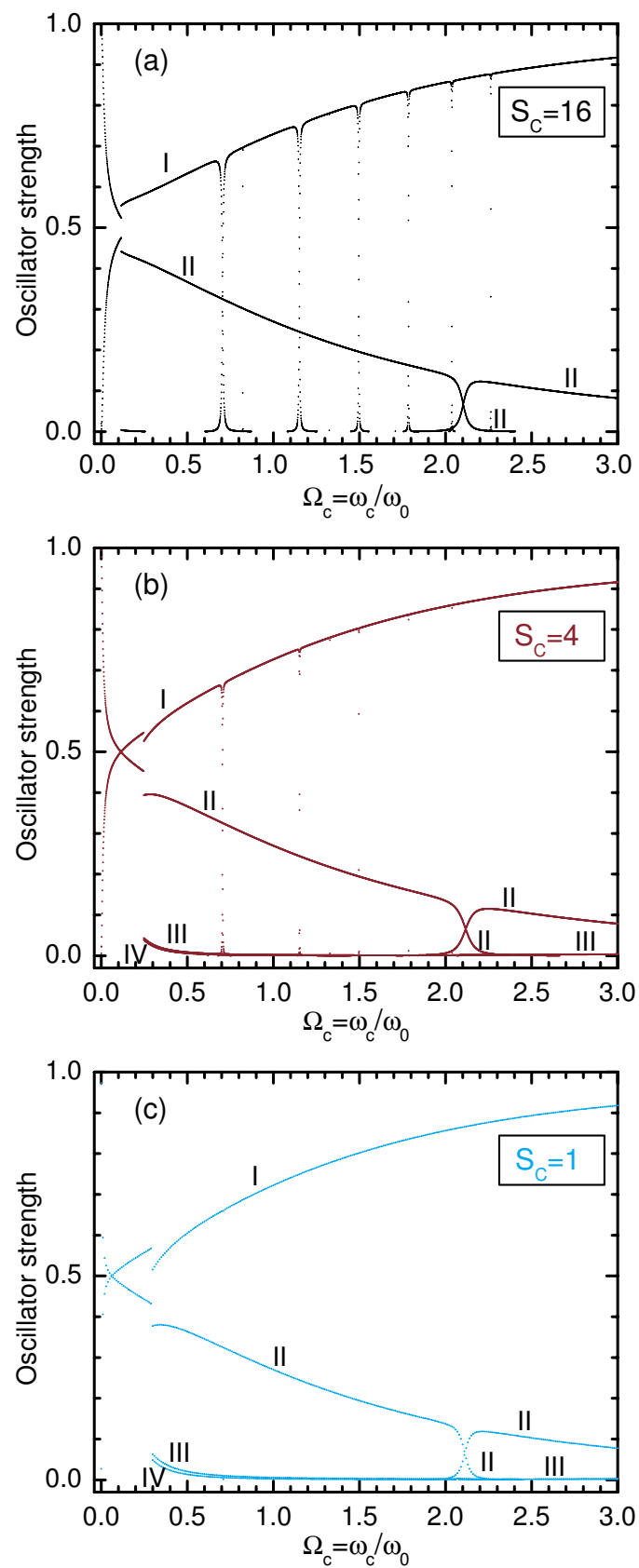

FIG. 12. (Color online) Oscillator strength with I, II, III, and IV each time indicating the four main transitions [two in (a)] corresponding to the four (three) branches in Fig. 11.

is closed for the case where the $\mathrm{Mn}$ ion is located at the center of the QD [see Fig. 10(a)]. This similar behavior takes place at other magnetic fields such as $\Omega_{c}=0.71$ [see Fig. 9(a)] and $\Omega_{c}=1.15$ [see Fig. 9(b)] for the other kind of electron transitions - the transition to the final state with negative azimuthal quantum number. This is due to the fact that around the above magnetic fields there exist two final states contributing to the electron transition with their wave functions composed of the two major FD states $(0,-1,1 / 2$, $-5 / 2)$ and $(0,0,1 / 2,-5 / 2)$. This results in a smaller region of the magnetic field as compared to the previous case around $\Omega_{c}=2.12$. 

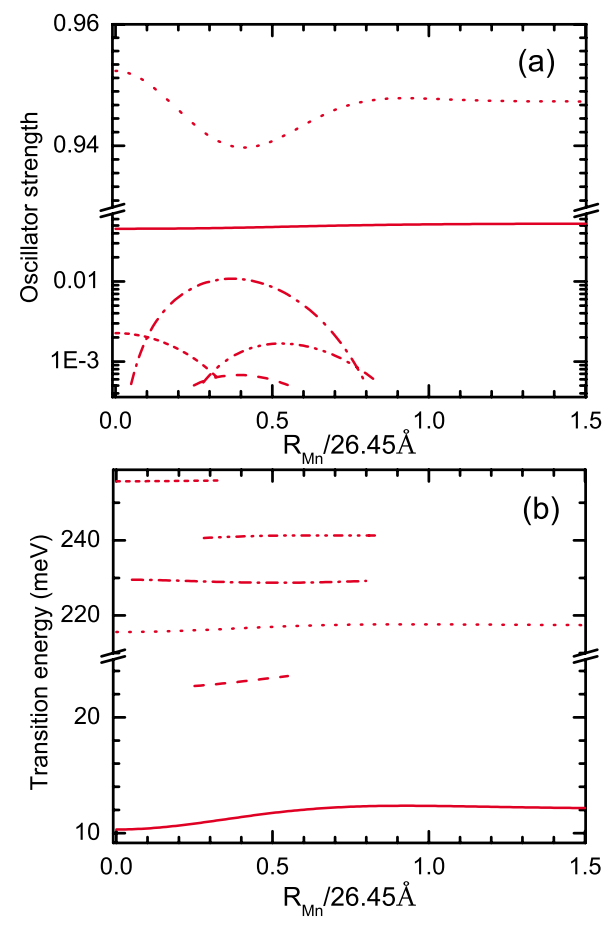

FIG. 13. (Color online) (a) The logarithm of the OS and (b) the transition energy vs the position of the magnetic ion at magnetic field $\Omega_{c}=4$ for confinement strength $S_{C}=4$. Solid and dotted lines are, respectively, the contributions from the second and the ninetyseventh levels as the main contributions to the electron transitions to the two $p$ orbitals.

Next we discuss the confinement-strength dependence of the electron transition first for the case where the Mn ion is located at the center of the quantum dot. We consider two different values of $S_{C}$, i.e., $S_{C}=4$ and 1 . The OS curve does not change with changing the confinement strength while the transition energy is proportional to $S_{C}$. This scaling is no longer exact if one displaces the $\mathrm{Mn}$ ion away from the center of the quantum dot, i.e., for $R_{\mathrm{Mn}}=(13.2 \AA, 0)=\left(0.5 l_{0}, 0\right)$, as can be seen from Fig. 11. The transition energies for three different $S_{C}=16,4$, and 1 that are all scaled to the case of $S_{C}=4$ now exhibit different behaviors as seen in Figs. 11(a)-11(c). First, the FM-AFM transition that leads to a discontinuity is found at different magnetic fields, i.e., $\Omega_{c}$ $=0.12,0.25$, and 0.3 for $S_{C}=16,4$, and 1, respectively. Besides, the anticrossing point at $\Omega_{c}=2.12$ as seen already from Fig. 9 is different for different $S_{C}$. It is now at 2.1, 2.12, and 2.11 for $S_{C}=16,4$, and 1, respectively. The OS also exhibits different features at the above magnetic fields as can be seen from Figs. 12(a)-12(c). As the electron is strongly confined, i.e., the case of $S_{C}=16$, the wave function becomes strongly localized and because the $\mathrm{Mn}$ ion is now located at $\left(0.5 l_{0}, 0\right)$, which is far enough from the center of the dot, the exchange interactions between the $p$ electron and the $\mathrm{Mn}$ ion reduce. Consequently, the effect of the e-Mn-ion interaction on the CR lines results in several anticrossings in the transition energy (crossings in the OS) as can be seen from Figs. 11(a) and 12(a). The branches I and II in all six figures from Fig. 11(a) to Fig. 12(c) refer to the transitions of the electron from
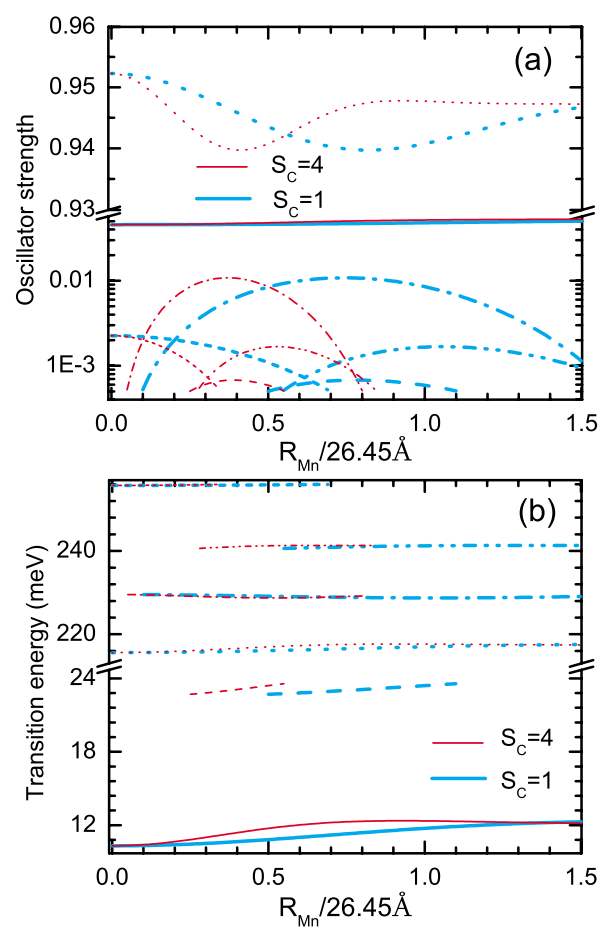

FIG. 14. (Color online) (a) The logarithm of the OS and (b) the transition energies of the same QD as plotted in Fig. 13 but for two different confinement strengths $S_{C}=4$ (six red thinner curves) and 1 (six cyan thicker curves). The final levels with the same relative position in the OS and two energy spectra (for two different $S_{C}$ ) have the same style of line.

the GS to the final states which have the FD states $(0$, $-1,1 / 2,-5 / 2)$ and $(0,1,1 / 2,-5 / 2)$ as the major contributions, respectively.

We now explore the magnetic-ion-position dependence of the OS within a large range of $R_{\mathrm{Mn}}$. We realize that at high magnetic field, the single-energy states "convert" to the Landau levels with the Landau indices $N_{L}=n+\frac{|l|-l+1}{2}$. We focus now on the high magnetic-field transitions for different positions of the Mn ion and different confinement strengths. As an example see Fig. 13, where at high magnetic field, e.g., $\Omega_{c}=4$, the electron transitions are mainly from the GS to the second (solid) and the ninety-seventh (dotted) levels; the upper part (dotted) of the OS curve oscillates and exhibits a maximum in the case where the $\mathrm{Mn}$ ion is at the center of the dot. In the latter case we have the largest contribution of the exchange energy to the total energy as compared to the other positions. Note also that if one keeps increasing the magnetic field, the small-contribution branches (dashed, dash dotted, dash dot dotted, and short dashed corresponding to the 5th, 98th, 101st, and 105th levels, respectively) in the OS curve, which are generally only about $2 \%-5 \%$ of their dominant branches (solid and dotted), become smaller. In the extremely high magnetic-field limit, the first electron transition, namely, to the energy level with the Fock-Darwin state $(0,1)$ being dominant, becomes zero making the transition to the level with the Fock-Darwin state $(0,-1)$ being dominant increases to unity. Therefore, the small-contribution branches in the OS curve plotted in Fig. 13(a) can be neglected in 
experimental measurements.

To complete this study we will examine the confinementstrength dependence of the electron transition for various positions of the Mn ion. It is understandable that for a stronger confinement, the electron becomes more localized resulting in a shifting of the OS curve to a smaller $R_{\mathrm{Mn}}$. This is shown in the OS behavior in Fig. 14(a) where the thinner OS curve (with six components) corresponds to the larger confinement strength $S_{C}=4$. For a less confined system (smaller value of $S_{C}$, i.e., $S_{C}=1$ ), the small-contribution branches are more expanded. With increasing $R_{\mathrm{Mn}}$, the problem converts to the problem without a magnetic ion. The small branches gradually disappear resulting in the saturation of the oscillator strength and transition energy for very large values of $R_{\mathrm{Mn}}$.

\section{DISCUSSIONS}

We studied the CR transition properties of a $\mathrm{Mn}^{2+}$-doped quantum dot containing a single electron confined by a parabolic potential in the presence of magnetic field. The transition energies and the oscillator strength were obtained for different positions of the magnetic ion with different confinement strengths.

As compared to the usual quantum dot (see, e.g., Ref. 19) without a magnetic ion, different transition frequencies are found to be allowed. These different transitions are due to the presence of the spin exchange interaction of the electron with the Mn ion which mixes the Fock-Darwin orbitals. Furthermore, the energy spectrum exhibits many anticrossings of energy levels that are not present in the usual single-electron parabolic quantum dot system.

At small magnetic fields the electron spin and the magnetic-ion spin are parallel which is similar to a FM state. With increasing magnetic field a transition takes place where the electron spin becomes parallel to the magnetic-field direction leading to an AFM state. This FM-AFM transition exhibits clear signatures in the CR absorption spectrum: (1) the CR transition energies are discontinuous, (2) the oscillator strength of the allowed transitions are discontinuous, and (3) the number of allowed transitions, i.e., the number of peaks in the CR absorption spectrum, is different in the FM and in the AFM states. The magnetic-field value at which the FM-AFM transition occurs depends on the position of the magnetic ion and on the confinement strength $S_{C}$ which also influences the $\mathrm{CR}$ absorption spectrum. Besides, the anticrossings in the energy spectrum, which are due to the spinspin exchange interactions, result in anticrossings in the transition energies and at the same time crossings in the oscillator strengths. This is due to the fact that around these anticrossings (in the energy spectrum), there are competitions between two energy levels as the major contribution to the electron transition. Both of these energy levels contribute about $40: 60 \%$ or $50: 50 \%$ to the transition, e.g., from the GS to the $p$ state with positive or negative azimuthal quantum number. The number and the positions of these anticrossings (crossings) change with changing the Mn-ion position and $S_{C}$.

At high magnetic field where the energy levels convert to Landau levels, we obtained the magnetic-ion-position dependence of the CR lines for different confinement strengths and investigated it for increasing confinement strength. The electron transition to higher energy levels exhibits an oscillatory behavior as a function of the position of the magnetic ion. However, there is one particular ion position, i.e., the center of the quantum dot, where the OS is independent of the confinement strength.

\section{ACKNOWLEDGMENTS}

We are thankful to A. Govorov for fruitful discussions. This work was supported by the FWO-Vl (Flemish Science Foundation), the EU Network of Excellence: SANDiE, and the Belgian Science Policy (IAP).

\footnotetext{
*nga.nguyen@ua.ac.be

†rancois.peeters@ua.ac.be

${ }^{1}$ J. K. Furdyna, J. Appl. Phys. 64, R29 (1988).

${ }^{2}$ D. L. Klein, R. Roth, A. K. L. Lim, A. P. Alivisatos, and P. L. McEuen, Nature (London) 389, 699 (1997).

${ }^{3}$ Y. F. Chen, J. H. Huang, W. N. Lee, T. S. Chin, R. T. Huang, F. R. Chen, J. J. Kai, and H. C. Ku, Appl. Phys. Lett. 90, 022505 (2007).

${ }^{4}$ K. Chang, J. B. Xia, and F. M. Peeters, Appl. Phys. Lett. 82, 2661 (2003).

${ }^{5}$ L. Besombes, Y. Léger, L. Maingault, D. Ferrand, H. Mariette, and J. Cibert, Phys. Rev. Lett. 93, 207403 (2004).

${ }^{6}$ A. O. Govorov, Phys. Rev. B 70, 035321 (2004); 72, 075358 (2005).

${ }^{7}$ Paul I. Archer, Steven A. Santangelo, and Daniel R. Gamelin, Nano Lett. 7, 1037 (2007).

${ }^{8}$ T. Schmidt, M. Scheibner, L. Worschech, A. Forchel, T. Slobodskyy, and L. W. Molenkamp, J. Appl. Phys. 100, 123109 (2006).
}

${ }^{9}$ P. Wojnar, J. Suffczyński, K. Kowalik, A. Golnik, G. Karczewski, and J. Kossut, Phys. Rev. B 75, 155301 (2007).

${ }^{10}$ J. Fernández-Rossier and Ramón Aguado, Phys. Rev. Lett. 98, 106805 (2007); J. Fernández-Rossier, Phys. Rev. B 73, 045301 (2006).

${ }^{11}$ Y. Léger, L. Besombes, J. Fernández-Rossier, L. Maingault, and H. Mariette, Phys. Rev. Lett. 97, 107401 (2006); L. Maingault, L. Besombes, Y. Léger, H. Mariette, and C. Bougerol, Phys. Status Solidi C 3, 3992 (2006); M. M. Glazov, E. L. Ivchenko, L. Besombes, Y. Léger, L. Maingault, and H. Mariette, Phys. Rev. B 75, 205313 (2007).

${ }^{12}$ I. Savić and N. Vukmirović, Phys. Rev. B 76, 245307 (2007).

${ }^{13}$ Shun-Jen Cheng, Phys. Rev. B 72, 235332 (2005); 77, 115310 (2008).

${ }^{14}$ F. Qu and P. Hawrylak, Phys. Rev. Lett. 95, 217206 (2005); 96, 157201 (2006).

${ }^{15}$ N. T. T. Nguyen and F. M. Peeters, Phys. Rev. B 76, 045315 (2007). 
${ }^{16}$ N. T. T. Nguyen and F. M. Peeters, Phys. Rev. B 78, 045321 (2008).

${ }^{17}$ W. Kohn and J. M. Luttinger, Phys. Rev. 96, 529 (1954); J. M. Luttinger, ibid. 102, 1030 (1956).

${ }^{18}$ Ch. Sikorski and U. Merkt, Surf. Sci. 229, 282 (1990); U. Merkt,
Phys. Rev. Lett. 76, 1134 (1996).

${ }^{19}$ F. Geerinckx, F. M. Peeters, and J. T. Devreese, J. Appl. Phys. 68, 3435 (1990).

${ }^{20}$ F. M. Peeters, Phys. Rev. B 42, 1486 (1990). 